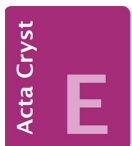

CRYSTALLOGRAPHIC COMMUNICATIONS

ISSN 2056-9890

Received 8 June 2015

Accepted 15 June 2015

Edited by M. Weil, Vienna University of Technology, Austria

Keywords: crystal structure; transition metal phosphates; alluaudite structure type; hydrothermal synthesis; hydrogen bonding

CCDC reference: 1406819

Supporting information: this article has

supporting information at journals.iucr.org/e

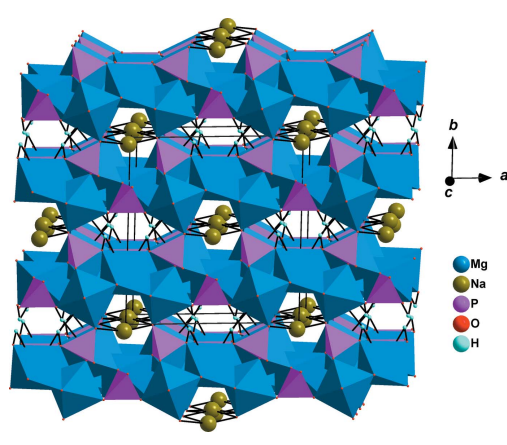

OPEN $\odot$ ACCESS

\section{Crystal structure of alluaudite-type $\mathrm{NaMg}_{3}\left(\mathrm{HPO}_{4}\right)_{2}\left(\mathrm{PO}_{4}\right)$}

\author{
Ahmed Ould Saleck, ${ }^{a, b *}$ Abderrazzak Assani, ${ }^{a}$ Mohamed Saadi, ${ }^{a}$ Cyrille Mercier, \\ Claudine Follet ${ }^{\mathrm{b}}$ and Lahcen El Ammari ${ }^{\mathrm{a}}$ \\ aLaboratoire de Chimie du Solide Appliquée, Faculté des Sciences, Université Mohammed V, Avenue Ibn Battouta, BP \\ 1014, Rabat, Morocco, and 'baboratoire des Matériaux Céramiques et Procédés Associés, EA2443, Université de \\ Valenciennes et du Hainaut-Cambrésis, Boulevard Charles de Gaulle, BP 59600, Maubeuge, France. *Correspondence \\ e-mail: a_ouldsaleck@yahoo.fr
}

The title compound, sodium trimagnesium bis(hydrogen phosphate) phosphate, was obtained under hydrothermal conditions. In the crystal, two types of $\left[\mathrm{MgO}_{6}\right]$ octahedra, one with point group symmetry 2, share edges to build chains

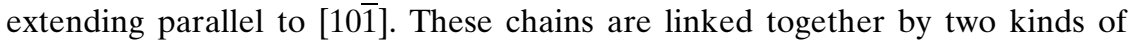
phosphate tetrahedra, $\mathrm{HPO}_{4}$ and $\mathrm{PO}_{4}$, the latter with point group symmetry 2 . The three-dimensional framework delimits two different types of channels extending along [001]. One channel hosts the $\mathrm{Na}^{+}$cations (site symmetry 2) surrounded by eight $\mathrm{O}$ atoms, with $\mathrm{Na}-\mathrm{O}$ bond lengths varying between 2.2974 (13) and 2.922 (2) $\AA$. The $\mathrm{OH}$ group of the $\mathrm{HPO}_{4}$ tetrahedron points into the other type of channel and exhibits a strong hydrogen bond to an $\mathrm{O}$ atom of the $\mathrm{PO}_{4}$ tetrahedron on the opposite side.

\section{Chemical context}

By means of hydrothermal processes (Demazeau, 2008; Yoshimura \& Byrappa, 2008), we have previously succeeded in the isolation of the mixed-valence manganese phosphates $M \mathrm{Mn}_{2}^{\mathrm{II}} \mathrm{Mn}^{\mathrm{III}}\left(\mathrm{PO}_{4}\right)_{3}(M=\mathrm{Ba}, \mathrm{Pb}, \mathrm{Sr})$ adopting the $\alpha-\mathrm{CrPO}_{4}$ structure type (Assani et al., 2013; Alhakmi et al., 2013a,b). In addition, within the pseudo-ternary systems $\mathrm{Ag}_{2} \mathrm{O}-\mathrm{MO}-\mathrm{P}_{2} \mathrm{O}_{5}$, hydrothermal syntheses have allowed us to obtain other $\alpha-\mathrm{CrPO}_{4}$ isotype phosphates, viz. $\mathrm{Ag}_{2} \mathrm{M}_{3}\left(\mathrm{HPO}_{4}\right)\left(\mathrm{PO}_{4}\right)_{2}$ $(M=\mathrm{Co}, \mathrm{Ni})$ while $\mathrm{AgMg}_{3}\left(\mathrm{HPO}_{4}\right)_{2}\left(\mathrm{PO}_{4}\right)$ is found to adopt the alluaudite structure type (Assani et al., 2011a,b,c). Other hydrothermally grown phosphates with the alluaudite structure include $\mathrm{AgCo}_{3}\left(\mathrm{HPO}_{4}\right)_{2}\left(\mathrm{PO}_{4}\right)$ (Guesmi \& Driss, 2002), $\mathrm{AgNi}_{3}\left(\mathrm{HPO}_{4}\right)_{2}\left(\mathrm{PO}_{4}\right)$ (Ben Smail \& Jouini, 2002), $A \mathrm{Mn}_{3}\left(\mathrm{HPO}_{4}\right)_{2}\left(\mathrm{PO}_{4}\right)(A=\mathrm{Na}, \mathrm{Ag})$ (Leroux et al., 1995a,b) and $\mathrm{NaCO}_{3}\left(\mathrm{HPO}_{4}\right)_{2}\left(\mathrm{PO}_{4}\right)$ (Lii \& Shih, 1994). Phosphates belonging to either the $\alpha-\mathrm{CrPO}_{4}$ or alluaudite structure type or derivatives thereof are still in the focus of research owing to their promising applications as battery materials ( $\operatorname{Trad} e t$ al., 2010; Essehli et al., 2015a,b; Huang et al., 2015).

The crystal structures of alluaudite-type phosphates exhibit channels in which the monovalent cations are localized. Indeed, this is strongly required for conductivity properties. The crystal structure of alluaudite can be formulated by the general formula $(A 1)(A 2)(M 1)(M 2)_{2}\left(\mathrm{PO}_{4}\right)_{3}$, (Moore \& Ito, 1979). The two $A$ sites can be occupied by either mono- or divalent medium-sized cations while the two $M$ cationic sites correspond to an octahedral environment generally occupied by transition metal cations. On the basis of literature research, it has been shown that the hydrothermal process allows, in general, stoechiometric phases to be obtained while solid-state 


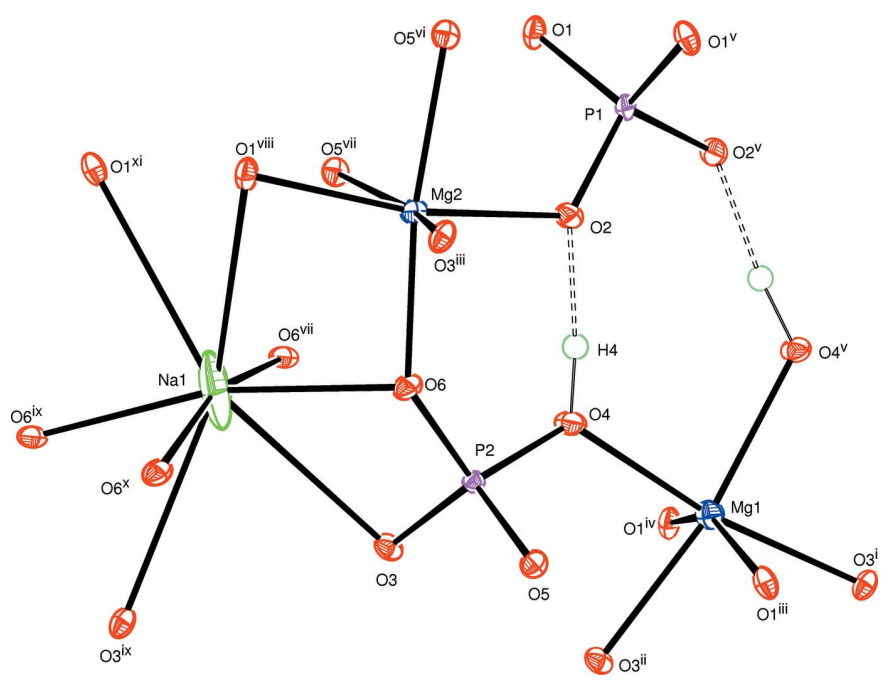

Figure 1

The principal building units in the structure of the title compound. Displacement ellipsoids are drawn at the $50 \%$ probability level. Hydrogen bonds are indicated by dashed lines [Symmetry codes: (i) $x+\frac{1}{2}, y+\frac{1}{2}, z$; (ii) $-x+\frac{3}{2}, y+\frac{1}{2},-z+\frac{1}{2}$; (iii) $-x+\frac{3}{2},-y+\frac{3}{2},-z+1$; (iv) $-x+\frac{3}{2}$, $-y+\frac{3}{2},-z$; (v) $-x+1,-y+1,-z$; (vi) $-x+1, y,-z+\frac{1}{2}$; (vii) $x,-y+1$, $z+\frac{1}{2}$; (viii) $x-\frac{1}{2},-y+\frac{3}{2}, z-\frac{1}{2}$; (ix) $-x+2, y,-z+\frac{3}{2}$; (x) $-x+2,-y+1$, $-z+1$; (xi) $x+\frac{1}{2},-y+\frac{1}{2}, z+\frac{1}{2}$; (xii) $-x+\frac{3}{2},-y+\frac{1}{2},-z+1$; (xiii) $x,-y+1$, $z-\frac{1}{2}$.]

reactions give rather a statistical distribution of cations on either the $A$ or $M$ sites, leading to non-stoechiometric compounds (Bouraima et al., 2015; Khmiyas et al., 2015).

In line with our focus of interest, we hydrothermally synthesized the compound $\mathrm{NaMg}_{3}\left(\mathrm{PO}_{4}\right)\left(\mathrm{HPO}_{4}\right)_{2}$ and report here its crystal structure.

\section{Structural commentary}

The principal building units of the allaudite structure of the title compound are represented in Fig. 1. The three atoms

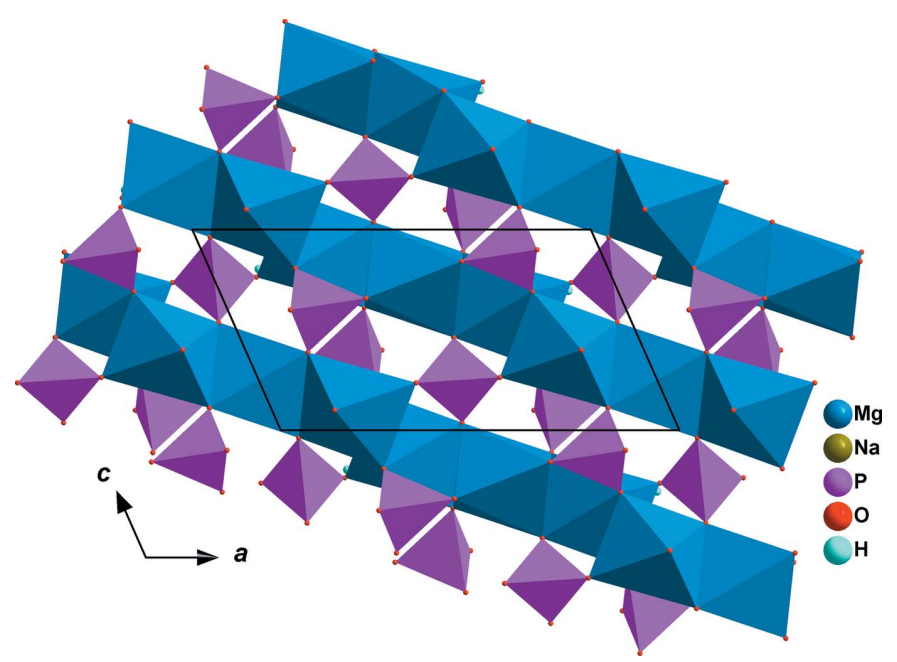

Figure 2

A sheet resulting from the linkage of kinked chains via vertices of $\mathrm{PO}_{4}$ tetrahedra.
Table 1

Selected bond lengths (®).

\begin{tabular}{llll}
\hline $\mathrm{Mg} 1-\mathrm{O}^{\mathrm{i}}$ & $2.1224(13)$ & $\mathrm{Na} 1-\mathrm{O} 3$ & $2.8840(19)$ \\
$\mathrm{Mg} 1-\mathrm{O} 1^{\mathrm{ii}}$ & $2.1312(12)$ & $\mathrm{Na} 1-\mathrm{O} 1^{\text {vii }}$ & $2.922(2)$ \\
$\mathrm{Mg} 1-\mathrm{O} 4$ & $2.1669(14)$ & $\mathrm{P} 1-\mathrm{O} 1^{\text {viii }}$ & $1.5372(12)$ \\
$\mathrm{Mg} 2-\mathrm{O} 6$ & $2.0234(13)$ & $\mathrm{P} 1-\mathrm{O} 1$ & $1.5372(12)$ \\
$\mathrm{Mg} 2-\mathrm{O} 3^{\text {ii }}$ & $2.0686(13)$ & $\mathrm{P} 1-\mathrm{O} 2^{\text {viii }}$ & $1.5476(13)$ \\
$\mathrm{Mg} 2-\mathrm{O} 2$ & $2.0696(14)$ & $\mathrm{P} 1-\mathrm{O} 2$ & $1.5476(13)$ \\
$\mathrm{Mg} 2-\mathrm{O}^{\text {iii }}$ & $2.0729(13)$ & $\mathrm{P} 2-\mathrm{O} 5$ & $1.5234(12)$ \\
$\mathrm{Mg} 2-\mathrm{O}^{\text {iv }}$ & $2.0955(13)$ & $\mathrm{P} 2-\mathrm{O} 6$ & $1.5263(12)$ \\
$\mathrm{Mg} 2-\mathrm{O}^{\mathrm{v}}$ & $2.1153(14)$ & $\mathrm{P} 2-\mathrm{O} 3$ & $1.5349(13)$ \\
$\mathrm{Na} 1-\mathrm{O}^{\mathrm{v}}$ & $2.2974(13)$ & $\mathrm{P} 2-\mathrm{O} 4$ & $1.5806(13)$ \\
$\mathrm{Na} 1-\mathrm{O}^{\text {vi }}$ & $2.4386(13)$ & & \\
\hline
\end{tabular}

Symmetry codes: (i) $x-\frac{1}{2},-y+\frac{1}{2}, z-\frac{1}{2}$; (ii) $x,-y+1, z-\frac{1}{2}$; (iii) $-x+\frac{1}{2}, y+\frac{1}{2},-z+\frac{1}{2}$; (iv) $x,-y+1, z+\frac{1}{2}$; (v) $-x+\frac{1}{2},-y+\frac{3}{2},-z+1$; (vi) $-x+1,-y+1,-z+1$; (vii) $x+\frac{1}{2},-y+\frac{3}{2}, z+\frac{1}{2}$; (viii) $-x, y,-z+\frac{1}{2}$.

Table 2

Hydrogen-bond geometry $\left(\AA{ }^{\circ}\right)$.

\begin{tabular}{lllll}
\hline$D-\mathrm{H} \cdots A$ & $D-\mathrm{H}$ & $\mathrm{H} \cdots A$ & $D \cdots A$ & $D-\mathrm{H} \cdots A$ \\
\hline $\mathrm{O} 4-\mathrm{H} 4 \cdots \mathrm{O} 2$ & 0.93 & 1.57 & $2.4932(17)$ & 174 \\
\hline
\end{tabular}

$\mathrm{Mg} 1, \mathrm{Na} 1$ and $\mathrm{P} 1$ are located on a twofold rotation axis (Wyckoff position 4e). Selected interatomic distances are compiled in Table 1. The three-dimensional framework of this structure consists of kinked chains of edge-sharing $\mathrm{MgO}_{6}$

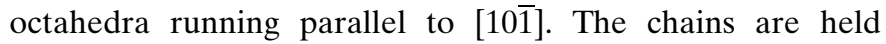
together by regular $\mathrm{P}_{1} \mathrm{O}_{4}$ phosphate groups, forming sheets perpendicular to [010], as shown in Fig. 2. The stacked sheets delimit two types of channels along [001]. One of the channels is occupied by $\mathrm{Na}^{+}$cations surrounded by eight oxygen atoms (Table 1), whereas the second channel contains the hydrogen atoms of the $\mathrm{HP}_{2} \mathrm{O}_{4}$ tetrahedra, as shown in Fig. 3. They form strong hydrogen bonds (Table 2, Figs. 1 and 3) with one of the oxygen atoms of $\mathrm{PO}_{4}$ tetrahedra on opposite sides.

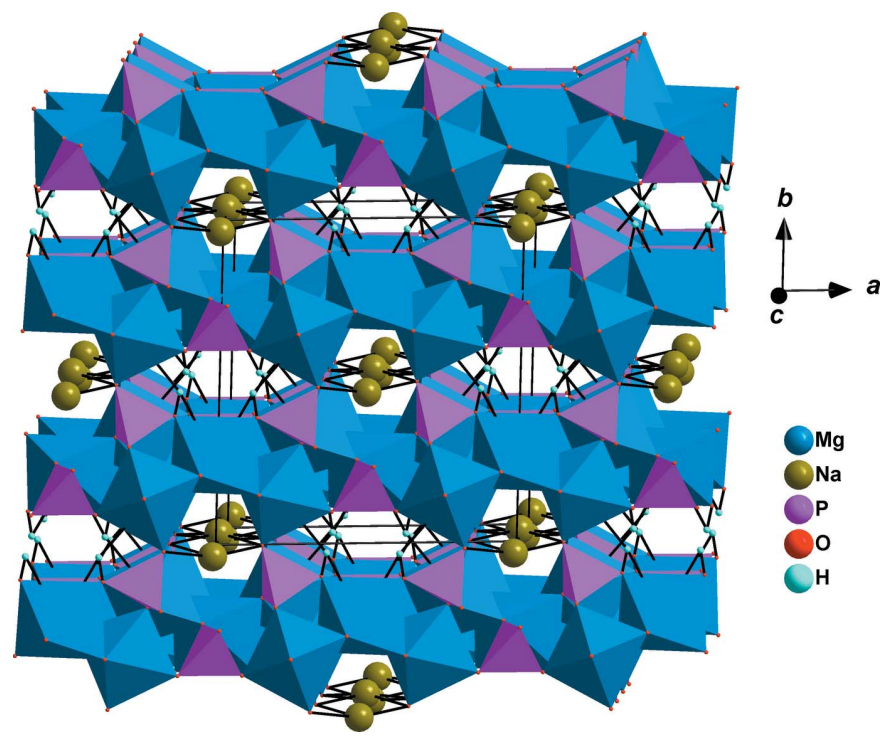

Figure 3

Polyhedral representation of the $\mathrm{NaMg}_{3}\left(\mathrm{HPO}_{4}\right)_{2}\left(\mathrm{PO}_{4}\right)$ structure showing channels along [001]. The $\mathrm{O}-\mathrm{H} \cdots \mathrm{O}$ hydrogen bonds are indicated by dashed lines. 
Table 3

Experimental details.

\begin{tabular}{|c|c|}
\hline \multicolumn{2}{|l|}{ Crystal data } \\
\hline Chemical formula & $\mathrm{NaMg}_{3}\left(\mathrm{HPO}_{4}\right)_{2}\left(\mathrm{PO}_{4}\right)$ \\
\hline$M_{\mathrm{r}}$ & 382.85 \\
\hline Crystal system, space group & Monoclinic, $C 2 / c$ \\
\hline Temperature $(\mathrm{K})$ & 296 \\
\hline$a, b, c(\AA)$ & $11.8064(6), 12.0625(7), 6.4969$ (4) \\
\hline$\beta\left(^{\circ}\right)$ & $113.805(2)$ \\
\hline$V\left(\AA^{3}\right)$ & $846.54(8)$ \\
\hline$Z$ & 4 \\
\hline Radiation type & Мо $K \alpha$ \\
\hline$\mu\left(\mathrm{mm}^{-1}\right)$ & 1.06 \\
\hline Crystal size $(\mathrm{mm})$ & $0.36 \times 0.24 \times 0.18$ \\
\hline \multicolumn{2}{|l|}{ Data collection } \\
\hline Diffractometer & Bruker X8 APEX \\
\hline Absorption correction & $\begin{array}{l}\text { Multi-scan (SADABS; Bruker, } \\
\text { 2009) }\end{array}$ \\
\hline$T_{\min }, T_{\max }$ & $0.504,0.748$ \\
\hline $\begin{array}{l}\text { No. of measured, independent and } \\
\text { observed }[I>2 \sigma(I)] \text { reflections }\end{array}$ & $9797,1291,1138$ \\
\hline$R_{\text {int }}$ & 0.038 \\
\hline$(\sin \theta / \lambda)_{\max }\left(\AA^{-1}\right)$ & 0.714 \\
\hline \multicolumn{2}{|l|}{ Refinement } \\
\hline$R\left[F^{2}>2 \sigma\left(F^{2}\right)\right], w R\left(F^{2}\right), S$ & $0.025,0.072,1.09$ \\
\hline No. of reflections & 1291 \\
\hline No. of parameters & 88 \\
\hline H-atom treatment & $\mathrm{H}$-atom parameters constrained \\
\hline$\Delta \rho_{\max }, \Delta \rho_{\min }\left(\mathrm{e} \AA^{-3}\right)$ & $0.57,-0.54$ \\
\hline
\end{tabular}

Computer programs: APEX2 and SAINT (Bruker, 2009), SHELXS (Sheldrick, 2008), SHELXL2013 (Sheldrick, 2015), ORTEP-3 for Windows (Farrugia, 2012). DIAMOND (Brandenburg, 2006) and publCIF (Westrip, 2010).

\section{Synthesis and crystallization}

Colourless parallelepiped-shaped crystals of the title compound were grown under hydrothermal conditions, starting from a mixture of $\mathrm{Na}_{4} \mathrm{P}_{2} \mathrm{O}_{7} \cdot 10 \mathrm{H}_{2} \mathrm{O}, \mathrm{MgO}$ and $\mathrm{H}_{3} \mathrm{PO}_{4}$ (85 wt \%) in the molar ratio $\mathrm{Na}_{4} \mathrm{P}_{2} \mathrm{O}_{7} \cdot 10 \mathrm{H}_{2} \mathrm{O}: \mathrm{MgO}: \mathrm{H}_{3} \mathrm{PO}_{4}=$ 1:3:3. The hydrothermal reaction was conducted in a $23 \mathrm{ml}$ Teflon-lined autoclave, filled to $50 \%$ with distilled water and under autogenous pressure at $483 \mathrm{~K}$ for four days.

\section{Refinement}

Crystal data, data collection and structure refinement details are summarized in Table 3 . The minimum and maximum electron densities are located 0.71 and $0.17 \AA$ from O5 and $\mathrm{H} 4$, respectively. The $\mathrm{O}-$ bound $\mathrm{H}$ atom was initially located in a difference map and refined with an $\mathrm{O}-\mathrm{H}$ distance restraint of $0.93 \AA$, and with $U_{\text {iso }}(\mathrm{H})=1.5 U_{\text {eq }}(\mathrm{O})$.

\section{Acknowledgements}

The authors thank the Unit of Support for Technical and Scientific Research (UATRS, CNRST) for the X-ray measurements and Mohammed V University, Rabat, Morocco, for financial support.

\section{References}

Alhakmi, G., Assani, A., Saadi, M. \& El Ammari, L. (2013a). Acta Cryst. E69, i40.

Alhakmi, G., Assani, A., Saadi, M., Follet, C. \& El Ammari, L. (2013b). Acta Cryst. E69, i56.

Assani, A., El Ammari, L., Zriouil, M. \& Saadi, M. (2011a). Acta Cryst. E67, i41.

Assani, A., El Ammari, L., Zriouil, M. \& Saadi, M. (2011b). Acta Cryst. E67, i40.

Assani, A., Saadi, M., Alhakmi, G., Houmadi, E. \& El Ammari, L. (2013). Acta Cryst. E69, i60.

Assani, A., Saadi, M., Zriouil, M. \& El Ammari, L. (2011c). Acta Cryst. E67, i5.

Ben Smail, R. \& Jouini, T. (2002). Acta Cryst. C58, i61-i62.

Bouraima, A., Assani, A., Saadi, M., Makani, T. \& El Ammari, L. (2015). Acta Cryst. E71, 558-560.

Brandenburg, K. (2006). DIAMOND. Crystal Impact GbR, Bonn, Germany.

Bruker (2009). APEX2, SAINT and SADABS. Bruker AXS Inc., Madison, Wisconsin, USA.

Demazeau, G. (2008). J. Mater. Sci. 43, 2104-2114.

Essehli, R., Belharouak, I., Ben Yahia, H., Chamoun, R., Orayech, B., El Bali, B., Bouziane, K., Zhoue, X. L. \& Zhoue, Z. (2015b). Dalton Trans. 44, 4526-4532.

Essehli, R., Belharouak, I., Ben Yahia, H., Maher, K., Abouimrane, A., Orayech, B., Calder, S., Zhou, X. L., Zhou, Z. \& Sun, Y.-K. (2015a). Dalton Trans 44, 7881-7886.

Farrugia, L. J. (2012). J. Appl. Cryst. 45, 849-854.

Guesmi, A. \& Driss, A. (2002). Acta Cryst. C58, i16-i17.

Huang, W., Li, B., Saleem, M. F., Wu, X., Li, J., Lin, J., Xia, D., Chu, W. \& Wu, Z. (2015). Chem. Eur. J. 21, 851-860.

Khmiyas, J., Assani, A., Saadi, M. \& El Ammari, L. (2015). Acta Cryst. E71, 690-692.

Leroux, F., Mar, A., Guyomard, D. \& Piffard, Y. (1995a). J. Solid State Chem. 117, 206-212.

Leroux, F., Mar, A., Payen, C., Guyomard, D., Verbaere, A. \& Piffard, Y. (1995b). J. Solid State Chem. 115, 240-246.

Lii, K.-H. \& Shih, P.-F. (1994). Inorg. Chem. 33, 3028-3031.

Moore, P. B. \& Ito, J. (1979). Mineral. Mag. 43, 227-35.

Sheldrick, G. M. (2008). Acta Cryst. A64, 112-122.

Sheldrick, G. M. (2015). Acta Cryst. C71, 3-8.

Trad, K., Carlier, D., Croguennec, L., Wattiaux, A., Ben Amara, M. \& Delmas, C. (2010). Chem. Mater. 22, 5554-5562.

Westrip, S. P. (2010). J. Appl. Cryst. 43, 920-925.

Yoshimura, M. \& Byrappa, K. (2008). J. Mater. Sci. 43, 2085-2103. 


\section{supporting information}

Acta Cryst. (2015). E71, 813-815 [doi:10.1107/S205698901501155X]

\section{Crystal structure of alluaudite-type $\mathrm{NaMg}_{3}\left(\mathrm{HPO}_{4}\right)_{2}\left(\mathrm{PO}_{4}\right)$}

\section{Ahmed Ould Saleck, Abderrazzak Assani, Mohamed Saadi, Cyrille Mercier, Claudine Follet and Lahcen El Ammari}

\section{Computing details}

Data collection: APEX2 (Bruker, 2009); cell refinement: SAINT (Bruker, 2009); data reduction: SAINT (Bruker, 2009); program(s) used to solve structure: SHELXS (Sheldrick, 2008); program(s) used to refine structure: SHELXL2013 (Sheldrick, 2015); molecular graphics: ORTEP-3 for Windows (Farrugia, 2012) and DIAMOND (Brandenburg, 2006); software used to prepare material for publication: publCIF (Westrip, 2010).

Sodium trimagnesium bis(hydrogen phosphate) phosphate

Crystal data

$\mathrm{NaMg}_{3}\left(\mathrm{HPO}_{4}\right)_{2}\left(\mathrm{PO}_{4}\right)$

$M_{r}=382.85$

Monoclinic, $C 2 / c$

$a=11.8064(6) \AA$

$b=12.0625(7) \AA$

$c=6.4969(4) \AA$

$\beta=113.805(2)^{\circ}$

$V=846.54(8) \AA^{3}$

$Z=4$

Data collection

Bruker X8 APEX

diffractometer

Radiation source: fine-focus sealed tube

Graphite monochromator

$\varphi$ and $\omega$ scans

Absorption correction: multi-scan

(SADABS; Bruker, 2009)

$T_{\min }=0.504, T_{\max }=0.748$

Refinement

Refinement on $F^{2}$

Least-squares matrix: full

$R\left[F^{2}>2 \sigma\left(F^{2}\right)\right]=0.025$

$w R\left(F^{2}\right)=0.072$

$S=1.09$

1291 reflections

88 parameters

0 restraints
$F(000)=760$

$D_{\mathrm{x}}=3.004 \mathrm{Mg} \mathrm{m}^{-3}$

Mo $K \alpha$ radiation, $\lambda=0.71073 \AA$

Cell parameters from 1291 reflections

$\theta=2.5-30.5^{\circ}$

$\mu=1.06 \mathrm{~mm}^{-1}$

$T=296 \mathrm{~K}$

Block, colourless

$0.36 \times 0.24 \times 0.18 \mathrm{~mm}$

9797 measured reflections

1291 independent reflections

1138 reflections with $I>2 \sigma(I)$

$R_{\text {int }}=0.038$

$\theta_{\max }=30.5^{\circ}, \theta_{\min }=2.5^{\circ}$

$h=-16 \rightarrow 16$

$k=-17 \rightarrow 17$

$l=-9 \rightarrow 8$

Hydrogen site location: difference Fourier map

$\mathrm{H}$-atom parameters constrained

$w=1 /\left[\sigma^{2}\left(F_{\mathrm{o}}^{2}\right)+(0.0362 P)^{2}+1.4203 P\right]$

where $P=\left(F_{\mathrm{o}}{ }^{2}+2 F_{\mathrm{c}}{ }^{2}\right) / 3$

$(\Delta / \sigma)_{\max }<0.001$

$\Delta \rho_{\max }=0.57 \mathrm{e} \AA^{-3}$

$\Delta \rho_{\min }=-0.54$ e $\AA^{-3}$ 
Special details

Geometry. All e.s.d.'s (except the e.s.d. in the dihedral angle between two l.s. planes) are estimated using the full covariance matrix. The cell e.s.d.'s are taken into account individually in the estimation of e.s.d.'s in distances, angles and torsion angles; correlations between e.s.d.'s in cell parameters are only used when they are defined by crystal symmetry. An approximate (isotropic) treatment of cell e.s.d.'s is used for estimating e.s.d.'s involving 1.s. planes.

Fractional atomic coordinates and isotropic or equivalent isotropic displacement parameters $\left(\AA^{2}\right)$

\begin{tabular}{lllll}
\hline & $x$ & $y$ & $z$ & $U_{\text {iso }} * / U_{\text {eq }}$ \\
\hline $\mathrm{Mg} 1$ & 0.0000 & $0.27947(7)$ & 0.2500 & $0.00857(18)$ \\
$\mathrm{Mg} 2$ & $0.29000(6)$ & $0.66219(5)$ & $0.37489(10)$ & $0.00643(14)$ \\
$\mathrm{Na} 1$ & 0.5000 & $0.52321(14)$ & 0.7500 & $0.0308(4)$ \\
$\mathrm{P} 1$ & 0.0000 & $0.68659(5)$ & 0.2500 & $0.00564(14)$ \\
$\mathrm{P} 2$ & $0.28093(4)$ & $0.38887(3)$ & $0.38603(7)$ & $0.00494(11)$ \\
O1 & $0.03662(11)$ & $0.75858(10)$ & $0.4624(2)$ & $0.0078(2)$ \\
O2 & $0.10795(12)$ & $0.61003(10)$ & $0.2634(2)$ & $0.0084(2)$ \\
O3 & $0.34567(12)$ & $0.32859(10)$ & $0.6116(2)$ & $0.0073(2)$ \\
O4 & $0.14051(11)$ & $0.40754(10)$ & $0.3420(2)$ & $0.0085(2)$ \\
H4 & 0.1241 & 0.4816 & 0.3033 & $0.013 *$ \\
O5 & $0.28443(11)$ & $0.32046(10)$ & $0.1916(2)$ & $0.0068(2)$ \\
O6 & $0.34273(12)$ & $0.50140(10)$ & $0.4000(2)$ & $0.0076(2)$ \\
& & & &
\end{tabular}

Atomic displacement parameters $\left(\AA^{2}\right)$

\begin{tabular}{lllllll}
\hline & $U^{11}$ & $U^{22}$ & $U^{33}$ & $U^{12}$ & $U^{13}$ & $U^{23}$ \\
\hline $\mathrm{Mg} 1$ & $0.0081(4)$ & $0.0088(4)$ & $0.0094(4)$ & 0.000 & $0.0041(3)$ & 0.000 \\
$\mathrm{Mg} 2$ & $0.0073(3)$ & $0.0057(3)$ & $0.0067(3)$ & $0.0004(2)$ & $0.0031(2)$ & $0.0001(2)$ \\
$\mathrm{Na} 1$ & $0.0118(6)$ & $0.0691(11)$ & $0.0091(6)$ & 0.000 & $0.0016(5)$ & 0.000 \\
$\mathrm{P} 1$ & $0.0051(3)$ & $0.0063(3)$ & $0.0044(3)$ & 0.000 & $0.0007(2)$ & 0.000 \\
$\mathrm{P} 2$ & $0.0060(2)$ & $0.00431(19)$ & $0.0043(2)$ & $-0.00006(14)$ & $0.00177(16)$ & $-0.00011(14)$ \\
$\mathrm{O} 1$ & $0.0063(6)$ & $0.0110(5)$ & $0.0054(5)$ & $-0.0010(4)$ & $0.0014(5)$ & $-0.0022(4)$ \\
$\mathrm{O} 2$ & $0.0060(6)$ & $0.0073(5)$ & $0.0114(6)$ & $0.0010(4)$ & $0.0031(5)$ & $-0.0007(4)$ \\
$\mathrm{O} 3$ & $0.0086(6)$ & $0.0080(5)$ & $0.0049(5)$ & $0.0020(4)$ & $0.0024(5)$ & $0.0016(4)$ \\
$\mathrm{O} 4$ & $0.0073(6)$ & $0.0058(5)$ & $0.0131(6)$ & $0.0009(4)$ & $0.0048(5)$ & $0.0004(5)$ \\
$\mathrm{O} 5$ & $0.0077(6)$ & $0.0077(5)$ & $0.0052(5)$ & $0.0003(4)$ & $0.0028(5)$ & $-0.0009(4)$ \\
$\mathrm{O} 6$ & $0.0083(6)$ & $0.0051(5)$ & $0.0093(6)$ & $-0.0014(4)$ & $0.0035(5)$ & $-0.0002(4)$ \\
\hline
\end{tabular}

Geometric parameters $\left(\AA,{ }^{o}\right)$

\begin{tabular}{llll}
\hline $\mathrm{Mg} 1-\mathrm{O}^{\mathrm{i}}$ & $2.1224(13)$ & $\mathrm{Na} 1-\mathrm{O} 6^{\mathrm{x}}$ & $2.4386(13)$ \\
$\mathrm{Mg} 1-\mathrm{O} 3^{\text {ii }}$ & $2.1224(13)$ & $\mathrm{Na}-\mathrm{O} 3$ & $2.8840(19)$ \\
$\mathrm{Mg} 1-\mathrm{O} 1^{\text {iii }}$ & $2.1312(12)$ & $\mathrm{Na} 1-\mathrm{O} 3^{\text {ix }}$ & $2.8840(19)$ \\
$\mathrm{Mg} 1-\mathrm{O} 1^{\text {iv }}$ & $2.1312(12)$ & $\mathrm{Na} 1-\mathrm{O} 1^{\mathrm{xi}}$ & $2.922(2)$ \\
$\mathrm{Mg} 1-\mathrm{O} 4^{\mathrm{v}}$ & $2.1669(14)$ & $\mathrm{Na}-\mathrm{O} 1^{\text {viii }}$ & $2.922(2)$ \\
$\mathrm{Mg} 1-\mathrm{O} 4$ & $2.1669(14)$ & $\mathrm{P} 1-\mathrm{O} 1^{\mathrm{v}}$ & $1.5372(12)$ \\
$\mathrm{Mg} 2-\mathrm{O} 6$ & $2.0234(13)$ & $\mathrm{P} 1-\mathrm{O} 1$ & $1.5372(12)$ \\
$\mathrm{Mg} 2-\mathrm{O} 3^{\text {iii }}$ & $2.0686(13)$ & $\mathrm{P} 1-\mathrm{O} 2^{\mathrm{v}}$ & $1.5476(13)$ \\
$\mathrm{Mg} 2-\mathrm{O} 2$ & $2.0696(14)$ & $\mathrm{P} 1-\mathrm{O} 2$ & $1.5476(13)$
\end{tabular}




\begin{tabular}{|c|c|c|c|}
\hline $\mathrm{Mg} 2-\mathrm{O}^{\mathrm{vi}}$ & $2.0729(13)$ & $\mathrm{P} 2-\mathrm{O} 5$ & $1.5234(12)$ \\
\hline $\mathrm{Mg} 2-\mathrm{O} 5^{\mathrm{vii}}$ & $2.0955(13)$ & $\mathrm{P} 2-\mathrm{O} 6$ & $1.5263(12)$ \\
\hline $\mathrm{Mg} 2-\mathrm{O} 1^{\mathrm{viii}}$ & $2.1153(14)$ & $\mathrm{P} 2-\mathrm{O} 3$ & $1.5349(13)$ \\
\hline $\mathrm{Na} 1-\mathrm{O} 6$ & $2.2974(13)$ & $\mathrm{P} 2-\mathrm{O} 4$ & $1.5806(13)$ \\
\hline $\mathrm{Na} 1-\mathrm{O}^{\mathrm{ix}}$ & $2.2974(13)$ & $\mathrm{O} 4-\mathrm{H} 4$ & 0.9269 \\
\hline $\mathrm{Na} 1-\mathrm{O}^{\mathrm{vii}}$ & $2.4386(13)$ & & \\
\hline $\mathrm{O}^{\mathrm{i}}-\mathrm{Mg} 1-\mathrm{O}^{\mathrm{ii}}$ & $104.23(8)$ & $\mathrm{O}^{\mathrm{vii}}-\mathrm{Na} 1-\mathrm{O}^{\mathrm{x}}$ & $166.01(10)$ \\
\hline $\mathrm{O}^{\mathrm{i}}-\mathrm{Mg} 1-\mathrm{O} 1^{\mathrm{iii}}$ & $86.53(5)$ & $\mathrm{O} 6-\mathrm{Na} 1-\mathrm{O} 3$ & $56.06(5)$ \\
\hline $\mathrm{O} 3^{\mathrm{ii}}-\mathrm{Mg} 1-\mathrm{O} 1^{\mathrm{iii}}$ & $78.23(5)$ & $\mathrm{O} 6^{\mathrm{ix}}-\mathrm{Na} 1-\mathrm{O} 3$ & $111.84(7)$ \\
\hline $\mathrm{O} 3^{\mathrm{i}}-\mathrm{Mg} 1-\mathrm{O} 1^{\mathrm{iv}}$ & $78.23(5)$ & $\mathrm{O} 6^{\mathrm{vii}-\mathrm{Na} 1-\mathrm{O} 3}$ & $62.51(5)$ \\
\hline $\mathrm{O}^{\mathrm{ii}}-\mathrm{Mg} 1-\mathrm{O}^{\mathrm{iv}}$ & $86.53(5)$ & $\mathrm{O} 6^{\mathrm{x}}-\mathrm{Na} 1-\mathrm{O} 3$ & $105.27(6)$ \\
\hline 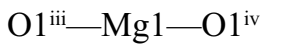 & $155.13(8)$ & $\mathrm{O} 6-\mathrm{Na} 1-\mathrm{O} 3^{\text {ix }}$ & $111.84(7)$ \\
\hline $\mathrm{O}^{\mathrm{i}}-\mathrm{Mg} 1-\mathrm{O}^{\mathrm{v}}$ & $83.70(5)$ & $\mathrm{O} 6^{\mathrm{ix}}-\mathrm{Na} 1-\mathrm{O} 3^{\mathrm{ix}}$ & $56.06(5)$ \\
\hline $\mathrm{O} 3^{\mathrm{ii}}-\mathrm{Mg} 1-\mathrm{O} 4^{\mathrm{v}}$ & $170.20(5)$ & 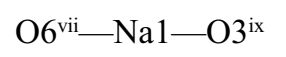 & $105.27(6)$ \\
\hline $\mathrm{O} 1^{\mathrm{iii}}-\mathrm{Mg} 1-\mathrm{O}^{\mathrm{v}}$ & $108.38(5)$ & $\mathrm{O} 6^{\mathrm{x}}-\mathrm{Na} 1-\mathrm{O} 3^{3^{\mathrm{x}}}$ & $62.51(5)$ \\
\hline $\mathrm{O} 1^{\mathrm{iv}}-\mathrm{Mg} 1-\mathrm{O} 4^{\mathrm{v}}$ & $89.53(5)$ & $\mathrm{O} 3-\mathrm{Na} 1-\mathrm{O} 3^{\text {ix }}$ & $71.02(6)$ \\
\hline $\mathrm{O}^{\mathrm{i}}-\mathrm{Mg} 1-\mathrm{O} 4$ & $170.20(5)$ & $\mathrm{O} 6-\mathrm{Na} 1-\mathrm{O} 1^{\mathrm{xi}}$ & $118.48(6)$ \\
\hline $\mathrm{O} 3^{\mathrm{ii}}-\mathrm{Mg} 1-\mathrm{O} 4$ & $83.70(5)$ & $\mathrm{O} 6^{\mathrm{ix}}-\mathrm{Na} 1-\mathrm{O} 1^{\mathrm{xi}}$ & $74.30(5)$ \\
\hline 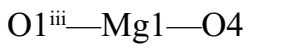 & $89.53(5)$ & 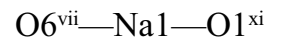 & $84.97(5)$ \\
\hline $\mathrm{O}^{\mathrm{iv}}-\mathrm{Mg} 1-\mathrm{O} 4$ & $108.38(5)$ & $\mathrm{O} 6^{\mathrm{x}}-\mathrm{Na} 1-\mathrm{O} 1^{\mathrm{xi}}$ & $107.88(6)$ \\
\hline $\mathrm{O} 4^{\mathrm{v}}-\mathrm{Mg} 1-\mathrm{O} 4$ & $89.05(7)$ & $\mathrm{O} 3-\mathrm{Na} 1-\mathrm{O} 1^{\mathrm{xi}}$ & $146.62(4)$ \\
\hline $\mathrm{O} 6-\mathrm{Mg} 2-\mathrm{O}^{\mathrm{iii}}$ & $85.88(5)$ & $\mathrm{O} 3^{\mathrm{ix}}-\mathrm{Na} 1-\mathrm{O} 1^{\mathrm{xi}}$ & $129.17(3)$ \\
\hline $\mathrm{O} 6-\mathrm{Mg} 2-\mathrm{O} 2$ & $88.80(5)$ & $\mathrm{O} 6-\mathrm{Na} 1-\mathrm{O} 1^{\mathrm{viii}}$ & $74.30(5)$ \\
\hline $\mathrm{O}^{\mathrm{iii}}-\mathrm{Mg} 2-\mathrm{O} 2$ & $111.03(6)$ & $\mathrm{O}^{\mathrm{ix}}-\mathrm{Na} 1-\mathrm{O} 1^{\text {viii }}$ & $118.48(7)$ \\
\hline $\mathrm{O} 6-\mathrm{Mg} 2-\mathrm{O}^{\mathrm{vi}}$ & $172.05(6)$ & $\mathrm{O}^{\mathrm{vii}}-\mathrm{Na} 1-\mathrm{O} 1^{\mathrm{viii}}$ & $107.88(6)$ \\
\hline $\mathrm{O}^{\mathrm{iii}}-\mathrm{Mg} 2-\mathrm{O}^{\mathrm{vi}}$ & $91.58(5)$ & $\mathrm{O} 6^{\mathrm{x}}-\mathrm{Na} 1-\mathrm{O} 1^{\mathrm{viii}}$ & $84.97(5)$ \\
\hline $\mathrm{O} 2-\mathrm{Mg} 2-\mathrm{O} 5^{\mathrm{vi}}$ & $85.07(5)$ & $\mathrm{O} 3-\mathrm{Na} 1-\mathrm{O}^{\text {viii }}$ & $129.17(3)$ \\
\hline $\mathrm{O} 6-\mathrm{Mg} 2-\mathrm{O}^{\mathrm{vii}}$ & $98.45(5)$ & $\mathrm{O} 3^{\text {ix }}-\mathrm{Na} 1-\mathrm{O} 1^{\text {viii }}$ & $146.62(4)$ \\
\hline $\mathrm{O} 3^{\mathrm{iii}}-\mathrm{Mg} 2-\mathrm{O} 5^{\mathrm{vii}}$ & $162.38(6)$ & $\mathrm{O} 1^{\mathrm{xi}}-\mathrm{Na} 1-\mathrm{O} 1^{\mathrm{vii}}$ & $51.46(6)$ \\
\hline $\mathrm{O} 2-\mathrm{Mg} 2-\mathrm{O} 5^{\mathrm{vii}}$ & $86.23(5)$ & $\mathrm{O} 1^{\mathrm{v}}-\mathrm{P} 1-\mathrm{O} 1$ & $111.21(10)$ \\
\hline $\mathrm{O} 5^{\mathrm{vi}}-\mathrm{Mg} 2-\mathrm{O} 5^{\mathrm{vii}}$ & $86.22(5)$ & $\mathrm{O} 1^{\mathrm{v}}-\mathrm{P} 1-\mathrm{O} 2^{\mathrm{v}}$ & $111.07(7)$ \\
\hline $\mathrm{O} 6-\mathrm{Mg} 2-\mathrm{O} 1^{\text {viii }}$ & $100.87(6)$ & $\mathrm{O} 1-\mathrm{P} 1-\mathrm{O} 2^{\mathrm{v}}$ & $108.34(7)$ \\
\hline $\mathrm{O} 3^{\mathrm{iii}}-\mathrm{Mg} 2-\mathrm{O} 1^{\mathrm{viii}}$ & $79.79(5)$ & $\mathrm{O} 1^{\mathrm{v}}-\mathrm{P} 1-\mathrm{O} 2$ & $108.34(7)$ \\
\hline $\mathrm{O} 2-\mathrm{Mg} 2-\mathrm{O} 1^{\text {viii }}$ & $166.18(6)$ & $\mathrm{O} 1-\mathrm{P} 1-\mathrm{O} 2$ & $111.07(7)$ \\
\hline $\mathrm{O} 5^{\text {vi }}-\mathrm{Mg} 2-\mathrm{O} 1^{\text {viii }}$ & $86.06(5)$ & $\mathrm{O} 2^{\mathrm{v}}-\mathrm{P} 1-\mathrm{O} 2$ & $106.73(10)$ \\
\hline $\mathrm{O} 5^{\mathrm{vii}}-\mathrm{Mg} 2-\mathrm{O} 1^{\text {viii }}$ & $82.62(5)$ & $\mathrm{O} 5-\mathrm{P} 2-\mathrm{O} 6$ & $111.03(7)$ \\
\hline $\mathrm{O} 6-\mathrm{Na} 1-\mathrm{O}^{6}{ }^{\mathrm{ix}}$ & $166.85(10)$ & $\mathrm{O} 5-\mathrm{P} 2-\mathrm{O} 3$ & $111.42(7)$ \\
\hline $\mathrm{O} 6-\mathrm{Na} 1-\mathrm{O}^{\mathrm{vii}}$ & $86.56(4)$ & $\mathrm{O} 6-\mathrm{P} 2-\mathrm{O} 3$ & $108.82(7)$ \\
\hline $\mathrm{O}^{\mathrm{ix}}-\mathrm{Na} 1-\mathrm{O} 6^{\mathrm{vii}}$ & $91.84(4)$ & $\mathrm{O} 5-\mathrm{P} 2-\mathrm{O} 4$ & $107.74(7)$ \\
\hline $\mathrm{O} 6-\mathrm{Na} 1-\mathrm{O}^{\mathrm{x}}$ & $91.84(4)$ & $\mathrm{O} 6-\mathrm{P} 2-\mathrm{O} 4$ & $108.99(7)$ \\
\hline $\mathrm{O}^{\mathrm{ix}}-\mathrm{Na} 1-\mathrm{O}^{\mathrm{x}}$ & $86.56(4)$ & $\mathrm{O} 3-\mathrm{P} 2-\mathrm{O} 4$ & $108.78(7)$ \\
\hline
\end{tabular}

Symmetry codes: (i) $x-1 / 2,-y+1 / 2, z-1 / 2$; (ii) $-x+1 / 2,-y+1 / 2,-z+1$; (iii) $x,-y+1, z-1 / 2$; (iv) $-x,-y+1,-z+1$; (v) $-x, y,-z+1 / 2$; (vi) $-x+1 / 2, y+1 / 2$, $-z+1 / 2$; (vii) $x,-y+1, z+1 / 2$; (viii) $-x+1 / 2,-y+3 / 2,-z+1$; (ix) $-x+1, y,-z+3 / 2$; (x) $-x+1,-y+1,-z+1$; (xi) $x+1 / 2,-y+3 / 2, z+1 / 2$. 
Hydrogen-bond geometry $\left(\AA,{ }^{\circ}\right)$

\begin{tabular}{lllll}
\hline$D-\mathrm{H} \cdots A$ & $D-\mathrm{H}$ & $\mathrm{H} \cdots A$ & $D \cdots A$ & $D-\mathrm{H} \cdots A$ \\
\hline $\mathrm{O} 4-\mathrm{H} 4 \cdots \mathrm{O} 2$ & 0.93 & 1.57 & $2.4932(17)$ & 174 \\
\hline
\end{tabular}

